\title{
Implantes de polietileno gel e poroso em cavidade anof tálmica de coelhos
}

\author{
Gel and porous polyethylene implants in the rabbitanophthalmiccavity
}

\author{
Lucieni Cristina Barbarini Ferraz ${ }^{1}$ \\ Silvana Artioli Schellini ${ }^{2}$ \\ Sheila Lordelo Wludarski ${ }^{3}$ \\ Carlos RobertoPadovani ${ }^{4}$
}

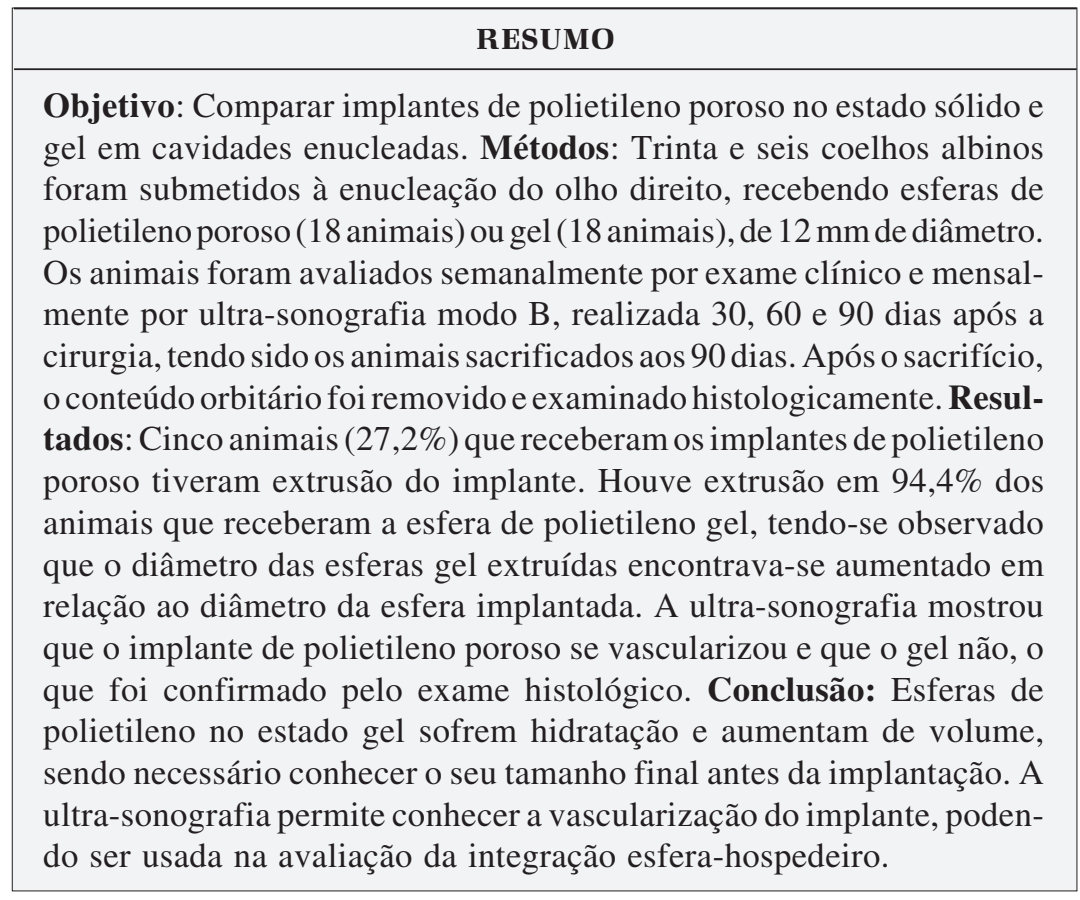

Descritores: Polietilenos/uso terapêutico; Implantes orbitários/métodos; Coelhos/cirurgia; Ultra-sonografia

autora e recebeu auxílio da CAPES para sua execução.

${ }^{1}$ Pós-Graduanda da Faculdade de Medicina da Universidade Estadual Paulista "Júlio Mesquita Filho" UNESP - Botucatu (SP) - Brasil.

${ }^{2}$ Livre-Docente, Professora do Departamento de Oftalmologia, Otorrinolaringologia e Cirurgia de Cabeça e Pescoço da Faculdade de Medicina da UNESP - Botucatu (SP) - Brasil.

${ }^{3}$ Médica Patologista - Consultoria em Patologia - Botucatu (SP) - Brasil.

${ }^{4}$ Professor Titular do Departamento de Bioestatística Instituto de Biociências - UNESP - Botucatu (SP) Brasil.

Endereço para correspondência: Silvana Artioli Schellini. Departamento de OFT/ORL/CCP - Faculdade de Medicina de Botucatu - UNESP - Botucatu (SP) CEP 18618-970

E-mail: sartioli@fmb.unesp.br

Recebido para publicação em 27.06.2005

Aprovação em 11.07.2005

Nota Editorial: Depois de concluída a análise do artigo sob sigilo editorial e com a anuência da Dra. Ana Estela Besteti Pires Ponce Sant'Anna sobre a divulgação de seu nome como revisora, agradecemos sua participação neste processo.

\section{INTRODUĈ̣̃O}

Os implantes são utilizados para reposição do conteúdo orbitário em cavidades anoftálmicas. Existem diversos tipos de implantes, tendo sido sugerido o uso de esferas integradas (hidroxiapatita, polietileno poroso), a partir da década de 80 do século passado ${ }^{(1)}$.

Com qualquer dos implantes integrados existentes, há possibilidade de se desenvolver deiscência conjuntival ${ }^{(2-3)}$. Várias são as causas para as deiscências, uma delas poderia ser ao fato de que os implantes se movimentam em conjunto com o conteúdo orbitário e as próteses externas poderiam ocasionar traumas repetidos para a superfície conjuntival ${ }^{(4)}$.

Esferas que não fossem constituídas de materiais rígidos poderiam ter vantagens neste sentido. Assim, idealizou-se uma esfera composta por uma substância no estado gelatinoso, composta de polietileno gel, o que deixaria o contato entre a mucosa conjuntival e a prótese externa menos traumático.

Os implantes integrados sofrem vascularização progressiva, resultando na integração entre a esfera e o hospedeiro. A avaliação desta integração foi sugerida utilizando-se métodos de custo elevado, como a tomografia
\end{abstract}


computadorizada ou a ressonância magnética ${ }^{(5-7)}$. Em estudo prévio, foi possível constatar que há possibilidade de se avaliar a integração das esferas por meio de exames ultra-sonográficos $^{(8)}$.

Desta forma, o objetivo deste estudo foi observar a resposta à implantação de esferas de polietileno poroso no estado sólido e gelatinoso ou gel em cavidades enucleadas de coelhos, pesquisando a integração esfera-hospedeiro por meio de ultra-sonografia modo B.

\section{MÉTODOS}

Trinta e seis coelhos foram submetidos a enucleação do olho direito, tendo sido implantadas esferas de polietileno poroso (Polipore - Homus Biotecnologia-SP, Brasil) em 18 animais (Grupo PP) e polietileno gel (Polietigel - Homus Biotecnologia-SP, Brasil) em 18 animais (Grupo PG). Todas as esferas eram de $12 \mathrm{~mm}$ de diâmetro, tendo sido feitas pelo mesmo profissional e eram constituídas basicamente pela mesma substância química. No entanto, diferiam pelo estado da substância, uma vez que as esferas de polietileno gel apresentavam a substância no estado gel, contida no formato desejado por estar envolta por uma membrana que a circundava em 360 graus.

Os coelhos foram submetidos a avaliações clínicas semanais, exame ultra-sonográfico modo B 30, 60 e 90 dias após o procedimento cirúrgico, tendo sido sacrificados aos 90 dias após a cirurgia. Após o sacrifício, o conteúdo orbitário foi removido e preparado para exame histológico, usando a coloração de hematoxilina e eosina.

Os resultados obtidos foram avaliados segundo estatística descritiva, usando a técnica da Freqüência de Ocorrência ${ }^{(9)}$.

\section{RESULTADOS}

\section{Avaliação clínica}

Grupo PP: houve extrusão da esfera de PP em cinco animais $(27,2 \%)$ que receberam implantes de polietileno poroso, o que ocorreu 30 dias após a cirurgia. Havia deiscência conjuntival prévia em 2 deles $(11,1 \%)$. Um dos coelhos deste grupo foi excluído por apresentar infecção sistêmica, não relacionada com o procedimento.

Grupo PG: houve extrusão da esfera de PG em 94,4\% dos animais que a receberam, ou seja, apenas uma esfera permaneceu na cavidade até os 90 dias. As extrusões ocorreram nos primeiros dias após a cirurgia, sendo o período de maior freqüência de ocorrência por volta de 20 dias. Observou-se que, embora as esferas de Polietigel implantadas tivessem $12 \mathrm{~mm}$ de diâmetro, no momento da extrusão apresentavam diâmetro ao redor de $20 \mathrm{~mm}$. Inflamação ou infecção orbitária não foram observadas nos animais que apresentaram extrusão da esfera.

\section{Avaliação ultra-sonográfica}

A avaliação pelo ultra-som modo B mostrou:

Grupo PP: vascularização dentro das esferas, com resposta do hospedeiro preenchendo os poros da esfera até a região central aos 30 dias após a cirurgia, com moderada refletividade (40-60\%). A refletividade se manteve até os 90 dias após a cirurgia, época em que a imagem se mostrou mais eco-densa, com estrutura interna do implante irregular e com atenuação acústica (Figura 1).

Grupo PG: as esferas de PG não se vascularizaram durante o período de seguimento. Houve baixa refletividade no interior das esferas e alta refletividade na porção posterior ao implante até os 90 dias após a cirurgia (Figura 2).

\section{Avaliação histológica}

Grupo PP: a avaliação histológica das esferas de PP mostrou fibroblastos e células inflamatórias no interior das esferas, de forma que, nos animais de 30 dias, estas células encon-

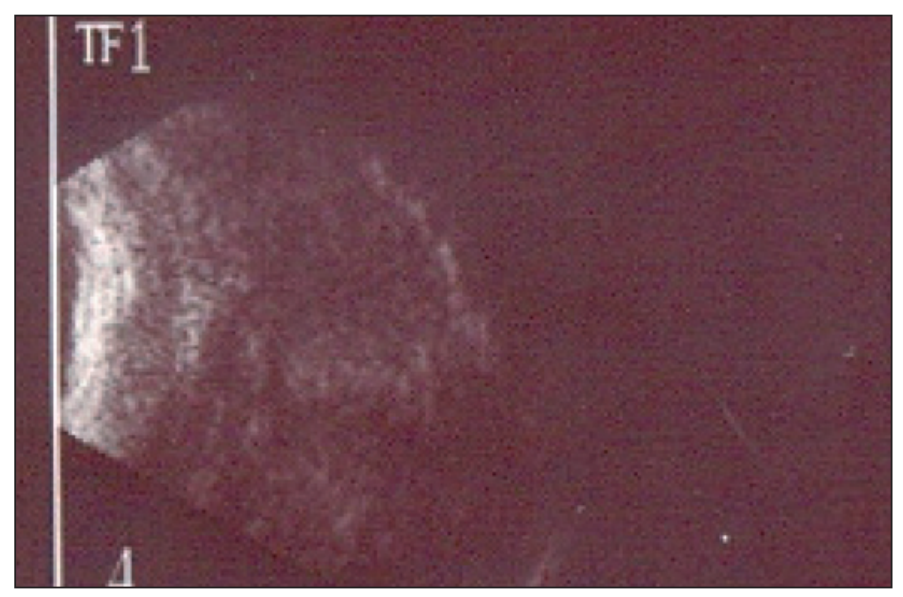

Figura 1 - Ecografia B de implante de polietileno poroso 90 dias após a implantação. Existe refletividade dentro da esfera.

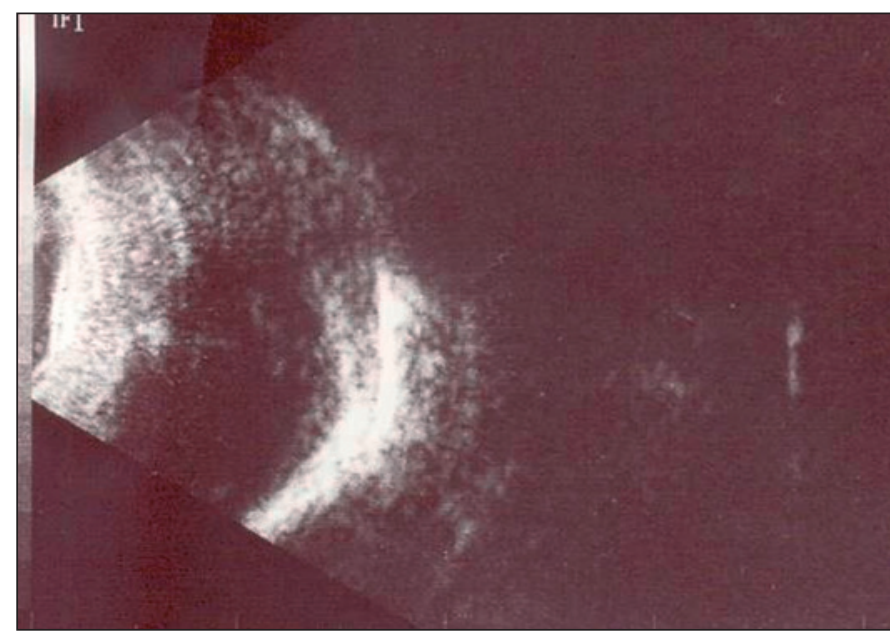

Figura 2 - Ecografia B de implante de polietileno gel 90 dias após a cirurgia. $O$ implante permaneceu com baixa refletividade até este momento experimental. Alta refletividade posteriormente ao implante. 
travam-se mais na periferia do implante, mas já alcançavam a área central. Nos de 60 dias, a esfera já possuía resposta fibrovascular em todo o seu interior e aos 90, a reação tecidual era mais densa. As esferas eram circundadas por uma pseudocápsula constituída por fibroblastos (Figura 3).

Grupo PG: houve somente uma esfera que chegou ao final do período idealizado para estudo. O exame histológico desta esfera mostrou material inerte, sem reação fibrovascular no seu interior. Ao redor desta esfera, observou-se pseudocápsula constituída por fibroblastos (Figura 4).

\section{DISCUSSÃO}

Este estudo comparou esferas de polietileno poroso no estado sólido e esferas de polietileno em sua forma gelatinosa. Embora as esferas possuíssem a mesma estrutura química, as

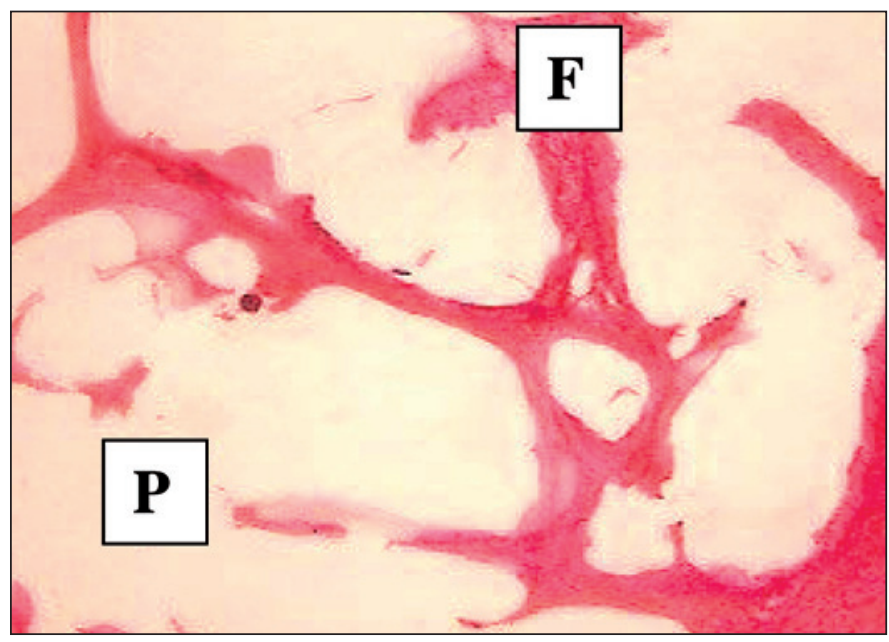

Figura 3 - Implante de polietileno poroso $(P)$ e reação tecidual como se fossem cordões de tecido fibrovascular $(F)$, preenchendo os espaços entre os grânulos do polietileno. Escassas células inflamatórias.

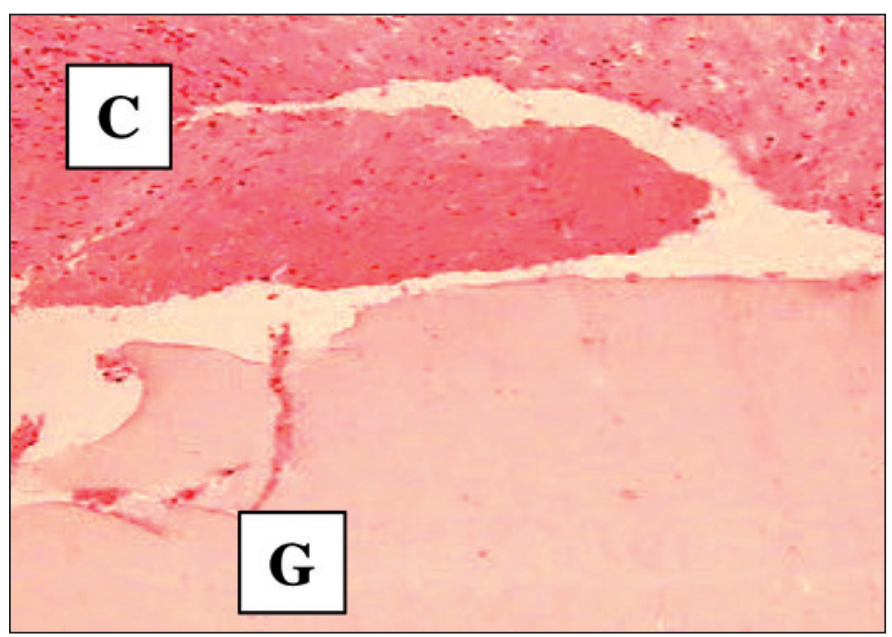

Figura 4 - Pseudocápsula (C) e o implante de polietileno gel (G), visibilizado como uma estrutura basofílica homogênea (HE x100) esferas de PG eram envoltas por uma membrana lisa, usada para conter o polietileno gel. Este implante não é rígido, como o polietileno poroso, tendo sido idealizado partindo-se do pressuposto de que as esferas rígidas poderiam ter um contato mais traumático com a prótese externa, o que favoreceria as deiscências e extrusões.

Porém, a avaliação clínica já mostrou que este tipo de implante deve sofrer modificações, uma vez que $94,4 \%$ das esferas sofreram extrusão, sem que houvessem sinais flogísticos. Além disso, as esferas apresentavam-se com diâmetro bastante aumentado no momento da expulsão. Desta forma, considerouse que a principal causa da extrusão do implante no estado gel foi o aumento de volume da esfera após a implantação, ocorrido, provavelmente, pela hidratação do material.

O implante aumentou em tamanho, pressionando e causando deiscência da sutura por força mecânica, o que resultou na expulsão do mesmo. Outro fato que reforça esta hipótese seria o período em que ocorreram as extrusões - logo nos primeiros dias após a implantação, sem que houvesse tempo para "rejeição" do material por fenômenos imunes.

Se o material se hidrata após a colocação, poderiam ser implantadas esferas menores. A colocação de esferas de diâmetro menor seria tecnicamente mais fácil, com necessidade de incisões menores. Porém, há necessidade de se conhecer o grau de hidratação da substância para que se tenha um controle do tamanho final que a esfera vai atingir quando estiver no seu estado definitivo.

A integração dos implantes com o hospedeiro pode ser atestada pelo grau de vascularização das esferas, comprovado por meio de métodos histológicos ${ }^{(10-12)}$ ou exames de imagem, como a tomografia ou a ressonância magnética ${ }^{(5-7)}$.

Por outro lado, pouco se comenta sobre a possibilidade de observar a vascularização no interior das esferas usando métodos não invasivos, como a ultra-sonografia, método já sugerido por outros ${ }^{(8)}$.

A vascularização do implante de polietileno poroso ocorre no primeiro mês de implantação ${ }^{(12-13)}$ e, por causa disso, no presente estudo optou-se por iniciar a avaliação ultra-sonográfica com 30 dias. Confirmando a eficiência do método, o uso desta técnica possibilitaria obter informações a respeito da esfera para estudos in vivo.

O implante de polietileno poroso, quando vascularizado, mostrou picos de refletividade baixa, semelhantes ao sangue $^{(14)}$, permitindo conhecer que o Polipore ${ }^{\circledR}$ recebe aumento gradual de neovasos e o Polietige ${ }^{\circledR}$ não se vasculariza. Portanto, este é um exame que pode ser incorporado às pesquisas como um dos indicativos de que o implante está recebendo vascularização, ou seja, está se integrando ao hospedeiro.

A avaliação histológica confirmou que o material recebe a resposta fibrovascular do hospedeiro e que existe a integração com as esferas de PP. A única esfera de PG estudada, não se vascularizou. A causa para isso poderia ser a membrana usada como envoltório da esfera gel que não foi efetiva para impedir sua hidratação, mas que impediu a integração tecidual que ocorre por células maiores e que não conseguiram transpor aquela membrana. 


\section{CONCLUSÕES}

Esferas em estado gel podem sofrer hidratação e aumentar de volume. Mais estudos devem ser feitos para determinar o volume final das esferas que se hidratam, para que se conheça qual seria o volume ideal da esfera a ser utilizada. A ultrasonografia pode ser utilizada como método indicativo da vascularização da esfera.

\section{ABSTRACT}

Purpose: To evaluate porous polyethylene implants as compared to solid and gel spheres in anophthalmic cavities. Methods: Thirty-six white rabbits underwent a right eye enucleation with placement of $12 \mathrm{~mm}$ of porous (18 animals) or gel (18 animals) polyethylene spheres. The animals were submitted to weekly clinical evaluation. Ultrasound examinations were done 30, 60 and 90 days after surgery and the animals were sacrificed on the day $90^{\text {th }}$. Then, the orbit content was removed and submitted to light microscopy. Results: Five animals $(27.2 \%)$ which received porous polyethylene spheres presented sphere extrusion. With the gel polyethylene spheres, the extrusions happened in $94.4 \%$ of the animals and it was observed that the expelled gel spheres had higher diameter then the implanted ones. Ultrasound evaluation showed that the porous polyethylene implant becomes vascularized but the gel not, as confirmed by histological examination. Conclusion: Spheres of material in the gel state hydrate and increase in volume making it necessary to know the final size before the implantation. Ultrasound examination allows the evaluation of the implant-tissue integration.
Keywords: Polyethylenes/therapeutic use; Orbital implants/ methods; Rabbits/surgery; Ultrasonography

\section{REFERÊNCIAS}

1. Karesh JW, Dresner SC. High-density porous polyethylene (Medpor) as a successful anophthalmic socket implant. Ophthalmology. 1994;101(10):168895; discussion 1695-6.

2. Shields CL, Shields JA, De Potter P. Hydroxyapatite orbital implant after enucleation. Experience with initial 100 consecutive cases. Arch Opthalmol. 1992;110(3):333-8

3. Shields CL, Shields JA, De Potter P, Singh AD. Problems with the hydroxyapatite orbital implant: experience with 250 consecutive cases. Br J Ophthalmol 1994;78(9):702-6

4. Schellini SA, Xavier AP, Hoyama E, Rossa R, Pellizon C, Marques ME, Padovani CR. Gelatinous polyethylene in the treatment of the anophthalmic cavity. Orbit. 2002;21(3):189-93.

5. Ainbinder DJ, Haik BG, Mazzoli RA. Anophthalmic socket and orbital implants Role of CT and MR imaging. Radiol Clin North Am. 1998;36(6):1133-47, xi.

6. De Potter P, Duprez T, Cosnard G. Postcontrast magnetic resonance imaging assessment of porous polyethylene orbital implant (Medpor). Ophthalmology. 2000;107(9):1656-60

7. Flanders AE, De Potter P, Rao VM, Tom BM, Shields CL, Shields JA. MRI of orbital hydroxyapatite implants. Neuroradiology. 1996;38(3):273-7.

8. Yamamoto ES, Hoyama E, Schellini SA, Padovani CR. Avaliação ultra-sonográfica do implante orbitário em cavidade anoftalmica. Rev Bras Oftalmol. 2001;60(9):637-42.

9. Norman GR, Streiner DL. Biostatistics: the bare essentials. St. Louis: Mosby; 1994.

10. Mawn LA, Jordan DR, Gilberg S. Proliferation of human fibroblasts in vitro after exposure to orbital implants. Can J Ophthalmol. 2001;36(5):245-51.

11. Rubin PA, Popham JK, Bilyk JR, Shore JW. Comparison of fibrovascular ingrowth into hydroxyapatite and porous polyethylene orbital implants. Ophthal Plast Reconstr Surg. 1994;10(2):96-103.

12. Schellini SA, Marques ME, Padovani CR, Taga EM, Rossa R. Comparison of synthetic hydroxyapatite and porous polyethylene implants in eviscerated rabbit eyes. Ophthal Plast Reconstr Surg. 2003;19(2):136-9.

13. Murray TG, Cicciarelli NL, Croft BH, Garonzik S, Voigt M, Hernandez E. Design of a magnetically integrated microporous implant. Arch Ophthalmol. 2000;118(9):1259-62.

14. Byrne SF, Green RL. Examination techniques for the orbit. In: Byrne SF, Green RL. Ultrasound of the eye and the orbit. St. Louis: Mosby; 2002. p.15-45;273-89.

\section{Congresso Cearense de Oftalmologia*}

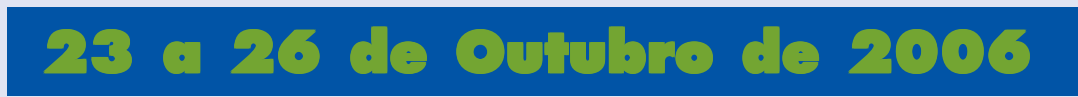

Fortaleza - CE

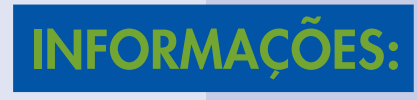

Tel.: (85) 4011-1572

E-mail: oftalmo@arxweb.com.br

*Participação neste evento conta 15 pontos para a revalidação do título de especialista em oftalmologia 